\title{
核磁共振技术测定联䒺酚的对映体纯度
}

\author{
奚忠华*，陆大东，戴洁，叶涛，章文伟 \\ 化学国家级实验教学示范中心(南京大学), 南京 210023
}

\begin{abstract}
摘要: 手性联菜酚(( $( \pm)-B I N O L)$ 制备是国内高校常开设的一个实验, 其产品的对映体纯度测试是实验的重要一环。以 2-甲酰基苯硼酸和 $(S)-(-)$-1-苯乙胺的混合物作为手性试剂与联菜酚发生Bull-James Assembly反应, 以此设计了核 磁共振氢谱 $\left({ }^{1} \mathrm{H} \mathrm{NMR}\right)$ 测定联菜酚对映体纯度的实验。实验结果表明, 通过反应生成的对映体混合物的核磁信号, 能 准确地计算出 $( \pm)-\mathrm{BINOL}$ 的对映体纯度。以选定的苯环氢核信号计算出的 $(R)-\mathrm{BINOL}$ 含量与理论ee值有着良好的线性 关系 $\left(R^{2}=0.9999\right)$ 。此实验方法能够快速完成大量学生样品的测量, 同时大大减少实验废液的产生量。学生通过此 实验可对核磁共振技术有更进一步的了解。
\end{abstract}

关键词：核磁共振；联菜酚；对映体纯度；对映体过量

中图分类号: G64; O6

\section{Determination of Enantiomeric Purity of Binaphthol by NMR Method}

\author{
Zhonghua Xi ${ }^{*}$, Dadong Lu, Jie Dai, Tao Ye, Wenwei Zhang \\ National Demonstration Center for Experimental Chemistry Education (Nanjing University), Nanjing 210023, China.
}

\begin{abstract}
The preparation of chiral binaphthol is a common experiment in colleges and universities, and the determination of enantiomeric purity is an important part of the experiment. The Bull-James Assembly between binaphthol and a mixture of 2-formylphenylboronic acid and (S)-(-)-1-phenylethylamine as chiral reagent was observed. Based on this, an experiment was designed to determine the enantiomer purity of binaphthol via ${ }^{1} \mathrm{H}$ NMR. The results show that the enantiomer purity of $( \pm)$-BINOL can be accurately calculated from the NMR signals of the enantiomer mixture generated by the reaction. There is a good linear relationship $\left(R^{2}=0.9999\right)$ between the $(R)$ BINOL ratio calculated from the selected proton signal and the given ee value. This method can quickly complete the measurement of a large number of student samples, and produce a small amount of waste liquid. Students can have a further understanding of NMR technology through this experiment.
\end{abstract}

Key Words: Nuclear magnetic resonance (NMR); Binaphthol (BINOL); Enantiomeric purity; Enantiomeric excess

在不对称合成和新药的研发过程中，测量手性化合物的纯度(对映体纯度)是一项必须的工作。 通常使用对映体过量(enantiomeric excess, 简称ee)来衡量手性化合物的纯度。实验室中测定ee值常用 的方法是手性固定相高效液相色谱法(chiral HPLC)。采用手性色谱方法来测定 $e e$ 值往往需要消耗大 量时间, 难于满足高通量的测试需求。核磁共振技术是鉴定有机化合物结构的有效手段, 采集一个 核磁氢谱消耗的时间小于五分钟。相较耗时的手性色谱方法, 核磁共振法可以更加快速地测定化合

收稿: 2021-09-10; 录用: 2021-09-13; 网络发表: 2021-11-05

“通讯作者, Email: xizh@nju.edu.cn

基金资助: 2021 年基础学科拔尖学生培养计划 2.0 课题 
物的ee值。

手性化合物中对映的氢核所产生的磁共振信号频率相同, 这意味着其在核磁共振谱图中的化学 位移相同, 因此无法通过核磁共振信号来区分对映体。通过添加手性试剂, 可以将对映体化合物转 变成非对映体的混合物, 混合物中原对映氢核若能产生适合分辨的信号, 则可以根据信号的强度来 确定原对映体化合物中对映体的含量, 从而得到ee值 ${ }^{[1]}$ 。

在医药工业中, 高纯度手性化合物有着广泛的应用, 因此手性合成是有机化学研究的热点领域 之一。在各种手性合成方法中, 不对称催化是一种非常高效的方法。以手性联菜酚 $(( \pm)-b i n a p h t h o l$, ( \pm -BINOL) (2) (图1)及其衍生物为配体的金属配合物是应用广泛的一类手性催化剂, 因而在教学中 开设手性联荎酚的制备实验 ${ }^{[2]}$ 是引入手性合成的有效手段, 目前全国很多高校都开设此实验。手性 联䒬酚制备完成后必须进行光学纯度的测定, 以检验合成方法的有效性。常见的测定方法有液相色 谱法 ${ }^{[3,4]}$ 、毛细管电泳法 ${ }^{[5]}$ 和核磁共振法 ${ }^{[6,7]}$, 本文对于手性联萗酚的 $e e$ 值测定采用简便的三组分手 性衍生方法 ${ }^{[8]}$ 。联菜酚与2-甲酰基苯嗍酸 (2-FPBA) (1)和 $(S)-(-)-1$-苯乙胺( $\left.(S)-\mathrm{MBA}\right)(3)$ 三者混合时会 发生Bull-James Assembly反应 ${ }^{[9]}$, 得到亚氨基硼酸酯(4)的非对映体混合物, 其中 $(R, S)-\mathbf{4}$ 和 $(S, S)-\mathbf{4}$ 的含 量比就对应了 ( $( \pm$ )-BINOL中不同构型的比例(图1)。这种手性衍生试剂核磁共振方法还可用于手性 1,2-, 1,3-，1,4-二醇和十多种手性伯胺的ee值测定 ${ }^{[10-12]}$ 。

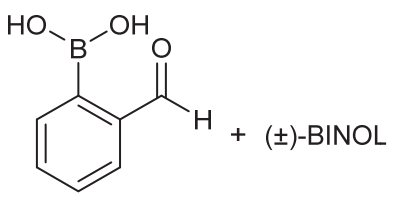

1

$( \pm)-2$
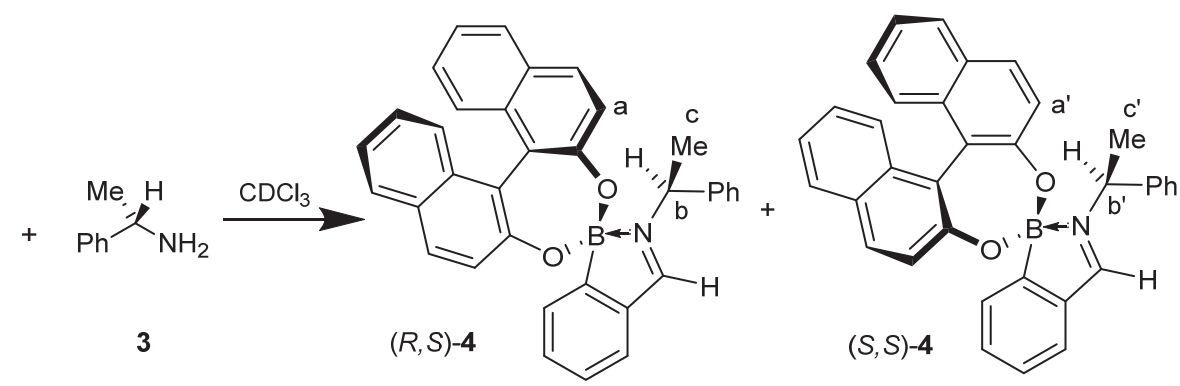

图1 测定联荎酚ee 值的Bull-James Assembly反应过程

\section{1 实验}

\section{1 仪器与试剂}

核磁共振波谱仪(布鲁克AVANCE III 400M，瑞士)，高效液相色谱仪(岛津LC20-AT，日本，二元 原, 紫外检测器), 纯水机(AWL-6000-U, 艾科浦), 移液器。

$2-F P B A(99.72 \%$, 上海皓鸿生物医药科技有限公司), $(S)-M B A(99 \%$, 萨恩化学技术有限公司 $)$, 氛代氯仿 $(99.8 \%, 0.03 \% \mathrm{TMS}$, 萨恩化学技术有限公司), $(R)$-BINOL $(99 \%$, 上海笛柏生物科技有限 公司), (S)-BINOL $(99 \%$, 上海笛柏生物科技有限公司), 联萗酚学生产品, 分子篮 $(4 \mathrm{~A}$ 型, 国药集团 化学试剂有限公司), 甲醇(HPLC级, TEDIA), 超纯水。

\section{2 样品准备}

分别准确称取 $29.0 \mathrm{mg}$ BINOL标准品 $((R)-\mathrm{BINOL}$ 含量范围为 $90 \%-10 \%)$ 和 $15.0 \mathrm{mg} 2-\mathrm{FPBA}$, 转移 至样品管中, 用移液器加入 $2.00 \mathrm{~mL}$ 気代氯仿溶解样品。将样品管放入温水浴中, 加快溶解速度。溶 解完成后, 加入 $4 \mathrm{~A}$ 分子篎5-6颗覆盖样品管底, 干燥, 待用, 记为溶液 A。学生产品采用同样操作步 骤。

准确称取 $37.0 \mathrm{mg}(S)-\mathrm{MBA}$ 于 $10.0 \mathrm{~mL}$ 样品管中, 用移液器加入 $5.00 \mathrm{~mL}$ 気代氯仿, 混合均匀后加 入 $4 \mathrm{~A}$ 分子笁5-6颗覆盖样品管底, 干燥, 待用, 记为溶液B。

\section{3 液相色谱分析}

用移液器移取干燥后的溶液 $\mathrm{A} 100 \mu \mathrm{L}$ 于 $2 \mathrm{~mL}$ 样品管中, 再加入 $900 \mu \mathrm{L}$ 甲醇稀释, 取 $5 \mu \mathrm{L}$ 进样分 
析。液相色谱分析条件如下: 采用菲罗门Chirex $(S)$-VAL and DNAn手性分离柱 $(4.6 \times 250 \mathrm{~mm}, 5 \mu \mathrm{m})$, 柱温 $40{ }^{\circ} \mathrm{C}$ ，流动相甲醇-水(体积比 $70: 30$ )，流速 $1.0 \mathrm{~mL} \cdot \mathrm{min}^{-1} ，$ 检测波长 $262 \mathrm{~nm}$ 。

\section{4 核磁分析}

用移液器分别移取干燥后的溶液 $\mathrm{A}$ 和溶液 $\mathrm{B}$ 各 $300 \mu \mathrm{L}$ 于同一核磁管内, 混合均匀后进行 ${ }^{1} \mathrm{H} N M R$ 测试。核磁谱图采集使用脉冲序列为 $z g$, 扫描次数为 16 次, 探头温度为 $297 \mathrm{~K}$, 谱宽 $20 \mathrm{ppm}$, 数据采 集使用Topspin 3.2, 核磁数据处理使用MestReNova 13.0。

\section{2 结果与讨论}

\section{1 对映体混合物核磁分析}

$(R, S)-\mathbf{4}$ 和 $(S, S)-\mathbf{4}$ 混合物核磁图谱如图2所示, 根据化学位移值和化合物结构可以看出混合物核磁 信号中, 有三组对映氢核的化学位移值可供确定 $( \pm)-B I N O L$ 的 $e e$ 值, 这三组氢核分别为苯环氢(a)、苄 基氢(b)和甲基氢(c)，其在化合物结构中的位置见图1标示，其化学位移值见表1。
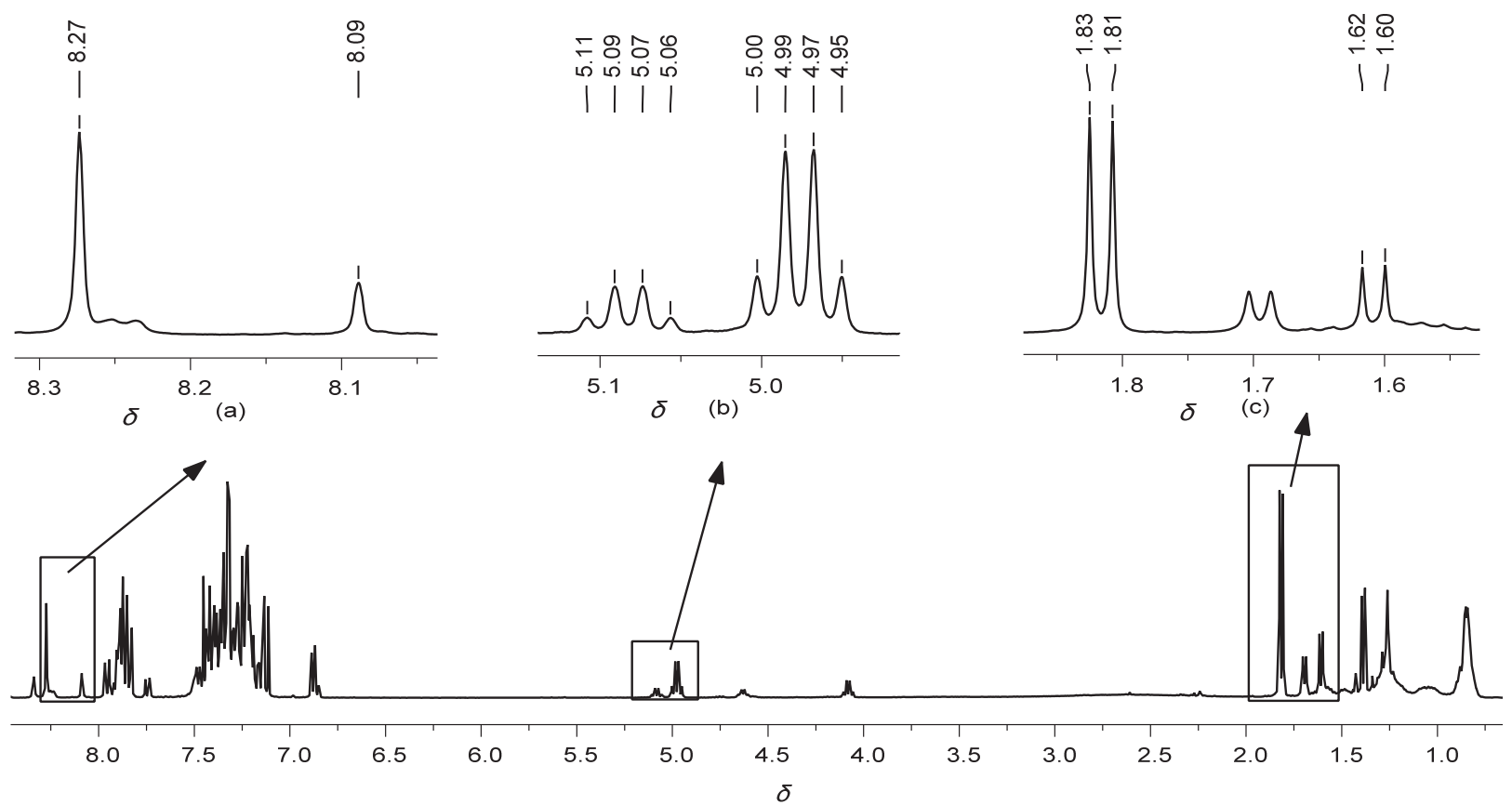

图 2 非对映体混合物 $400 \mathrm{MHz}{ }^{1} \mathrm{H}$ NMR谱图

溶剂: 氛代氯仿

表1 对映体混合物核磁共振化学位移

\begin{tabular}{ccc}
\hline 化合物 & 位置 & 化学位移 $\left(\delta_{\mathrm{H}}\right)$ \\
\hline$(R, S)-\mathbf{4}$ & $\mathrm{a}$ & $8.27(\mathrm{~s}, 1 \mathrm{H})$ \\
$(R, S)-\mathbf{4}$ & $\mathrm{b}$ & $4.97(\mathrm{q}, J=7.0 \mathrm{~Hz}, 1 \mathrm{H})$ \\
$(R, S)-\mathbf{4}$ & $\mathrm{c}$ & $1.82(\mathrm{~d}, 3 \mathrm{H})$ \\
$(S, S)-\mathbf{4}$ & $\mathrm{a}^{\prime}$ & $8.09(\mathrm{~s}, 1 \mathrm{H})$ \\
$(S, S)-\mathbf{4}$ & $\mathrm{b}^{\prime}$ & $5.08(\mathrm{q}, J=6.9 \mathrm{~Hz}, 1 \mathrm{H})$ \\
$(S, S)-\mathbf{4}$ & $\mathrm{c}^{\prime}$ & $1.61(\mathrm{~d}, 3 \mathrm{H})$ \\
\hline
\end{tabular}




\section{2 标准样品数据处理}

利用核磁法进行定量分析时可以采用峰高或峰面积, 本实验中采用峰高, 可避免其它信号的干 扰, 相比峰面积结果更优。联䒬酚标准品共准备9份, ee值范围为 $+80 \%--80 \%$, 使用 1.4 小节中方法 测试得到对映体混合物 $\mathbf{4}$ 的核磁数据, 根据表 1 的位置标注化学位移, 记录下对应的峰高, 根据三组 对映氢核的信号分别计算 $(R)-\mathrm{BINOL}$ 的含量 $(R \%)$, 计算结果见表 2 。 $(R)-\mathrm{BINOL}$ 的含量 $(R \%)$ 及其 $e e$ 值 计算公式如下:

$$
\begin{aligned}
& R \%=\frac{H_{R}}{H_{R}+H_{S}} \times 100 \% \\
& e e(R, \%)=\frac{H_{R}-H_{S}}{H_{R}+H_{S}} \times 100 \%
\end{aligned}
$$

其中, $H_{R}$ 为 $(R, S)-4$ 结构中 $\mathrm{a} 、 \mathrm{~b} 、 \mathrm{c}$ 位置处质子在 ${ }^{1} \mathrm{H}$ NMR谱图中的对应峰高, $H s$ 为 $(S, S)-4$ 结构中 $a^{\prime} 、 b^{\prime} 、 c^{\prime}$ 位置处质子在 ${ }^{1} H$ NMR谱图中的对应峰高。

对映体混合物液相色谱谱图数据处理时, 也采用上述公式, 只需将公式中的峰高替换为样品对 应的峰面积即可, 计算结果一并列入表2。

\begin{tabular}{|c|c|c|c|c|c|c|c|c|c|c|}
\hline \multirow{2}{*}{ No. } & \multirow{2}{*}{$R: S$} & \multirow{2}{*}{$e e$} & \multicolumn{2}{|c|}{ HPLC } & \multicolumn{2}{|c|}{${ }^{1} \mathrm{H}$ NMR, a } & \multicolumn{2}{|c|}{${ }^{1} \mathrm{H}$ NMR, b } & \multicolumn{2}{|c|}{${ }^{1} \mathrm{H}$ NMR, c } \\
\hline & & & $R \%$ & $e e$ & $R \%$ & $e e$ & $R \%$ & $e e$ & $R \%$ & $e e$ \\
\hline 1 & $90: 10$ & $80 \%$ & $88.9 \%$ & $77.8 \%$ & $88.6 \%$ & $77.2 \%$ & $88.7 \%$ & $77.5 \%$ & $87.8 \%$ & $75.5 \%$ \\
\hline 2 & $80: 20$ & $60 \%$ & $79.3 \%$ & $58.6 \%$ & $78.9 \%$ & $57.8 \%$ & $79.6 \%$ & $59.3 \%$ & $77.6 \%$ & $55.2 \%$ \\
\hline 3 & $70: 30$ & $40 \%$ & $68.1 \%$ & $36.2 \%$ & $68.4 \%$ & $36.8 \%$ & $69.6 \%$ & $39.3 \%$ & $66.4 \%$ & $32.8 \%$ \\
\hline 4 & $40: 60$ & $20 \%$ & $59.2 \%$ & $18.4 \%$ & $59.0 \%$ & $18.0 \%$ & $59.4 \%$ & $18.8 \%$ & $55.6 \%$ & $11.3 \%$ \\
\hline 5 & $50: 50$ & $0 \%$ & $49.7 \%$ & $-0.6 \%$ & $49.3 \%$ & $-1.4 \%$ & $49.6 \%$ & $-0.7 \%$ & $45.9 \%$ & $-8.2 \%$ \\
\hline 6 & $40: 60$ & $-20 \%$ & $39.8 \%$ & $-20.4 \%$ & $39.3 \%$ & $-21.4 \%$ & $40.4 \%$ & $-19.1 \%$ & $37.5 \%$ & $-25.0 \%$ \\
\hline 7 & $30: 70$ & $-40 \%$ & $29.9 \%$ & $-40.2 \%$ & $29.4 \%$ & $-41.2 \%$ & $29.3 \%$ & $-41.4 \%$ & $26.6 \%$ & $-46.7 \%$ \\
\hline 8 & $20: 80$ & $-60 \%$ & $19.9 \%$ & $-60.2 \%$ & $19.8 \%$ & $-60.4 \%$ & $20.3 \%$ & $-59.4 \%$ & $17.9 \%$ & $-64.1 \%$ \\
\hline 9 & $10: 90$ & $-80 \%$ & $9.7 \%$ & $-80.6 \%$ & $9.9 \%$ & $-80.2 \%$ & $9.9 \%$ & $-80.3 \%$ & $8.4 \%$ & $-83.2 \%$ \\
\hline
\end{tabular}

表2 联萗酚手性液相色谱方法与核磁共振方法 $(R)$-BINOL含量和 $e e$ 值测定结果

\section{3 定量分析对映氢核的选择}

以标准品中 $(R)-\mathrm{BINOL}$ 的理论 $e e$ 值和实际测量的百分含量作图，考查其线性关系，如图3所示， 该图采用苯环氢核磁信号计算样品含量, $R^{2}$ 为 0.9999 , 具有很好的线性关系。液相色谱方法和芐基氢 核磁信号两者的 $R^{2}$ 均为 0.9998 , 甲基氢 $R^{2}$ 为 0.9988 。

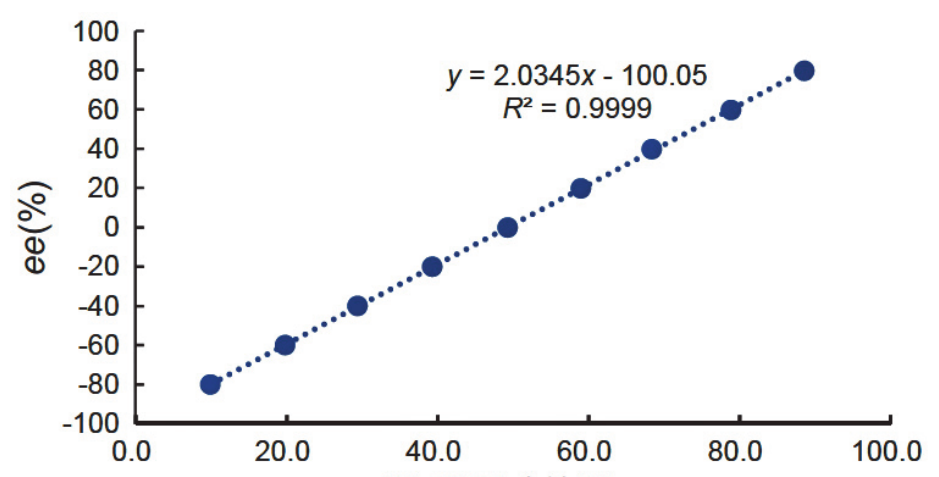

$(R)$-BINOL含量 $(\%)$

图3 标准样品理论ee值与 $(R)-B I N O L$ 测试含量标准曲线(苯环氢) 
采用甲基氢信号计算样品含量相对误差比苯环氢和苄基氢要大很多, 可能原因是甲基氢化学位 移处信号复杂, 基线难于处理平整, 导致峰高计算误差较大, 因此不宜采用此处信号作为定量标准。 苄基氢的核磁信号能很好地反映出标样中 $(R)$-BINOL的含量, 结果与苯环氢相近, 考虑到该处为四重 峰, 而苯环氢的核磁信号为单峰, 便于数据处理, 因此, 优先选择苯环氢信号计算样品含量。

\section{4 学生样品测试}

选取拆分前后的联菜酚学生样品, 按照标样测试方法进行测试, 选取液相色谱和苯环氢信号进 行数据处理, 结果见表 3 。手性液相色谱方法测量 $e e$ 值是目前公认的准确方法, 因此测试结果以该方 法所测得 $e e$ 值为参照考查核磁方法的可靠性, 由结果可知两种方法有着较好的一致性。表 3 中学生 1 号 样品核磁结果为 $(R)$-BINOL含量 $97.5 \%$, 此时 $(S)$-BINOL含量为 $2.5 \%$, 其信号信噪比为 $S N=10$, 可见 若样品中 $(R)-\mathrm{BINOL}$ 或 $(S)-\mathrm{BINOL}$ 含量大于 $97.5 \%$ 时, 核磁方法将无法提供准确的结果。

\section{表3 联菜酚学生样品测试结果}

\begin{tabular}{|c|c|c|c|c|c|}
\hline \multirow{2}{*}{ No. } & \multicolumn{2}{|c|}{ HPLC } & \multicolumn{2}{|c|}{${ }^{1} \mathrm{H}$ NMR } & \multirow{2}{*}{$e e$ 差值 } \\
\hline & $R \%$ & $e e$ & $R \%$ & $e e$ & \\
\hline 1 & 96.8 & $93.6 \%$ & 97.5 & $95.0 \%$ & $1.4 \%$ \\
\hline 2 & 12.2 & $-75.6 \%$ & 10.9 & $-78.2 \%$ & $-2.6 \%$ \\
\hline 3 & 48.6 & $-2.8 \%$ & 47.5 & $-5.0 \%$ & $-2.2 \%$ \\
\hline
\end{tabular}

\section{5 相比手性液相色谱方法的优劣性}

由前面实验数据可以看出核磁方法测定联菜酚对映体纯度是一个切实可行的方法。相比液相色 谱方法, 核磁方法有以下两个优点: 一是测量速度快, 一个样品测试约需 $5 \mathrm{~min}$, 而联荎酚手性液相 色谱分析约需 $10 \mathrm{~min}$, 其他手性样品可能需半小时以上; 二是试剂消耗大大减少, 液相色谱方法每测 一个样品会产生至少 $20 \mathrm{~mL}$ 废液, 而核磁方法只需要消耗约 $3 \mathrm{~mL}$ 试剂, 大大降低了废液的产生量。

手性液相色谱的关键主要在于手性色谱柱的选择, 目前商品化的手性柱能够针对不同类别的化 合物提供非常多的色谱柱选择, 而核磁技术测定对映体纯度关键在于手性试剂的选择, 针对不同待 测物需要寻找不同的手性试剂, 相比液相色谱方法普适性要差, 本文所用方法仅适合部分手性二醇 和手性伯胺的ee值测定。

核磁共振方法因为信噪比的问题, 其在检测高纯度物质时, 难于检测到微弱的杂质信号, 因此 该方法ee值测量结果有效性范围为 $-95.0 \%-95.0 \%$ 。另外, 在样品前处理方面, 核磁方法相对液相色 谱方法更加繁琐耗时。

在有机化学教学实验 “手性联荎酚的制备” 的样品表征中引入核磁的方法进行样品分析, 让学 生可以和经典的液相色谱法进行效果比较, 找出两种方法的优劣性, 从而拓宽其知识面。

\section{3 结语}

本文采用在联菜酚样品中添加2-甲酰基苯嗍酸和 $(S)-(-)-1$-苯乙胺的混合物作为手性试剂的方 法, 成功利用核磁共振氢谱技术测定了联荎酚的对映体纯度。在一定的 $e e$ 值范围内 $(-95.0 \%-95.0 \%)$, 与手性液相色谱方法相比, 核磁方法具有很好的准确性且分析速度快。学生通过分析非对映体混合 物复杂的核磁谱图, 可以进一步熟悉手性化合物结构并能深入了解核磁原理, 能提升学生的基本科 研素养。该方法适合作为有机化学教学实验 “手性联萗酚的制备” 的样品表征手段。

\section{参 考 文 献}


[2] 吴美芳, 吴琳. 有机化学实验. 北京: 科学出版社, 2013: 345-349.

[3] 林桂就, 韦志明, 穆允玲, 黄平, 黄科润. 化工技术与开发, 2013, 42 (6), 33.

[4] 王秧年，陆大东，奚忠华，叶涛，章文伟，李琳，张剑荣. 实验技术与管理，2015，32 (6), 168.

[5] 郑一宁, 谢天尧, 莫金垣, 韦寿莲. 应用化学, 2002, 18 (2), 113.

[6] 刘军, 王敏, 陈万义, 沈其丰. 波谱学杂志, 1996, 13 (4), 347.

[7] 王秀珍, 许遵乐. 化学研究, 2007, 18 (3), 21.

[8] Kelly, A. M.; Pérez-Fuertes, Y.; Arimori, S.; Bull, S. D.; James, T. D. Org. Lett. 2006, 8 (10), 1971.

[9] Pérez-Fuertes, Y.; Kelly, A. M.; Johnson, A. L.; Arimori, S.; Bull, S. D.; James, T. D. Org. Lett. 2006, 8 (4), 609.

[10] Kelly, A. M.; Pérez-Fuertes, Y.; Fossey, J. S.; Yeste, S. L.; Bull, S. D.; James, T. D. Nat. Protoc. 2008, 3 (2), 215.

[11] Brittain, W. D.; Chapin, B. M.; Zhai, W.; Lynch, V. M.; Buckley, B. R.; Anslyn, E. V.; Fossey, J. S. Org. Biomol. Chem. 2016, 14 (46), 10778.

[12] Fossey, J. S.; Anslyn, E. V.; Brittain, W. D. G.; Bull, S. D.; Chapin, B. M.; Le Duff, C. S.; James, T. D.; Lees, G.; Lim, S.; Lloyd, J. A. C.; et al. J. Chem. Educ. 2016, 94 (1), 79. 